\title{
Characterization of the Metabolic Fate of Datura metel Seed Extract and Its Main Constituents in Rats
}

\author{
Cong Xia ${ }^{1+}$, Yan $\mathrm{Liu}^{2 \dagger}$, Hai Qi ${ }^{3}$, Lulu Niu ${ }^{1}$, Yuxuan Zhu ${ }^{1}$, Wanying Lu ${ }^{1}$, Xinyi Xu ${ }^{1}$, \\ Yongjian Su ${ }^{1}$, Bingyou Yang ${ }^{2 *}$ and Qi Wang ${ }^{1 *+}$
}

\begin{abstract}
'Department of Medicinal Chemistry and Natural Medicine Chemistry, College of Pharmacy, Harbin Medical University, Harbin, China, ${ }^{2}$ Key Laboratory of Chinese Materia Medica, Heilongjiang University of Chinese Medicine, Harbin, China,

${ }^{3}$ Department of Cardiology, The 2nd Affiliated Hospital of Harbin Medical University, Harbin, China
\end{abstract}

\section{OPEN ACCESS}

Edited by:

Haitao Lu,

Shanghai Jiao Tong University, China

Reviewed by:

Eva Maria Pferschy-Wenzig,

University of Graz, Austria

Johanna Mahwahwatse Bapela,

University of Pretoria, South Africa

*Correspondence:

Bingyou Yang

ybywater@163.com

Qi Wang

mydearmumu@163.com

${ }^{t}$ These authors have contributed equally to this work

Specialty section:

This article was submitted to

Ethnopharmacology,

a section of the journal

Frontiers in Pharmacology

Received: 30 November 2018 Accepted: 06 May 2019

Published: 28 May 2019

Citation:

Xia C, Liu Y, Qi H, Niu L, Zhu Y,

Lu W, Xu X, Su Y, Yang B and Wang $Q$

(2019) Characterization of the

Metabolic Fate of Datura metel Seed

Extract and lts Main Constituents

in Rats. Front. Pharmacol. 10:571.

doi: 10.3389/fphar.2019.00571
Datura mete/ L. has been frequently used in Chinese traditional medicine. However, little is known on the chemical composition and in vivo metabolism of its seeds. In this study, using the strategy "chemical analysis, metabolism of single representative compounds, and metabolism of extract at clinical dosage" that we propose here, 42 constituents were characterized from $D$. metel seeds water extract. Furthermore, the metabolic pathways of 13 representative bioactive compounds of $D$. metel seeds were studied in rats after the oral administration of $D$. metel seeds water extract at a clinical dosage $(0.15 \mathrm{~g} / \mathrm{kg})$. These included three withanolides, two withanolide glucosides, four amides, one indole, one triterpenoid, one steroid, and one sesquiterpenoid, and with regard to phase II metabolism, hydroxylation, (de)methylation, and dehydrogenation reactions were dominant. Furthermore, the metabolism of $D$. metel seeds water extract provided to rats at a clinical dosage was investigated by liquid chromatography-tandem mass spectrometry based on the above metabolic pathways. Sixty-one compounds were detected in plasma, 83 in urine, and 76 in fecal samples. Among them, withanolides exhibited higher plasma exposure than the other types. To our knowledge, this is the first systematic study on the chemical profiling and metabolite identification of $D$. metel seeds, including all compounds instead of single constituents.

Keywords: Datura metel seeds, metabolites identification, LC-MS fingerprinting, withanolides, amides, indoles

\section{INTRODUCTION}

Datura metel L. (Solanaceae) seeds are one of the most popular herbal medicines for the treatment of rheumatoid arthritis and convulsions (Murthy et al., 2004). Although the seeds are toxic, they also have strong analgesic, anthelmintic, antioxidant, antimicrobial, antiviral, and antidiabetic activities (Wannang and Ndukwe, 2009; Kamaraj et al., 2011; Gu et al., 2014; Bachheti et al., 2018; Roy et al., 2018). Withanolides, flavonoids, alkaloids, sesquiterpenoids, lignans, and phenolic acids are generally considered the major bioactive compounds of D. metel (Kuang et al., 2008; Mai et al., 2017). However, there are few reports on the chemical composition of D. metel seeds. In previous studies, amides, indoles, sesquiterpenes, withanolides, and withanolide glucosides have been isolated from D. metel (Yang et al., 2010a,b; Bellila et al., 2011), but the constituents responsible for the treatment of different conditions have not been clarified. 
It is well known that the in vivo metabolites of herbal medicines may play a substantial role in the therapeutics. Therefore, metabolites' identification is critical for elucidating the bioactivities of complex herbal medicines. Recently, we studied the metabolism of two typological components, including two amides ( $n$-trans-feruloyltyramine and cannabisin F) and two withanolide glucosides (daturataturin A and daturametelin I) of D. metel seeds in rats (Xu et al., 2018). To fully predict and identify their metabolites, we selected seven main types of representative compounds from the seeds.

In this study, we aimed to improve this strategy so that it can be applied to other herbal medicines. Focusing on the identification of the in vivo metabolites of $D$. metel seed extracts after oral administration in rats, we first established the chemical fingerprint of $D$. metel seeds and identified 42 seed components (Figure 1). Second, we examined the in vivo metabolic pathways of seven groups of metabolites with different scaffolds, which included 13 representative compounds, using quadrupole time-of-flight mass spectrometry (qTOF-MS) and liquid chromatography-tandem mass spectrometry (LC/MS/MS). Finally, a normal clinical dosage of the herbal extract was administered to rats, and several metabolites were detected based on the metabolic pathways. Following this strategy, 113 metabolites (including 42 original phytochemicals and 71 newly formed ones) were detected at a clinical dosage $(0.15 \mathrm{~g} / \mathrm{kg})$ in rats.

\section{MATERIALS AND METHODS}

\section{Chemicals and Reagents}

The pure compounds daturametelindole B (1), daturametelin M (3), hyoscyamilactol (5), daturametelindole A (9), daturametelin L (29), daturametelindole $\mathrm{D}$ (18), n-trans-feruloyltyramine (19), daturametelindole C (22), daturametelin I (30), cannabisin F (31), daturaolone (32), daturataturin A (36), $n$-trans-p-coumaroyltyramine (40), and stigmasterol (41) were isolated from the seeds of $D$. metel by the authors. Their structures were characterized using nuclear magnetic resonance and MS (Figure 2 and Supplementary Figures S3-S22;

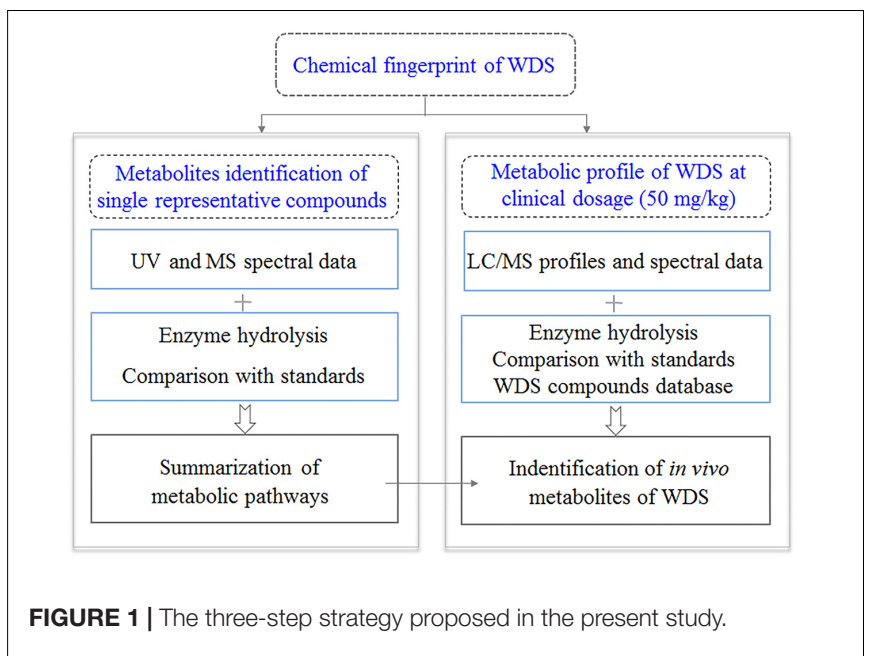

Xu et al., 2018). Dinoxin B (10), withametelin L (13), and cinerolide (20) were purchased from Nantong Feiyu Biological Technology Co., Ltd. (Nanjing, China). Purities were above $98 \%$ according to high-performance liquid chromatography-ultraviolet (HPLC/UV) analysis. Betaglucuronidase (HP-2 type, containing 100,000 U $\beta$-glucuronidase and $7500 \mathrm{U}$ sulfatase per milliliter), $\beta$-nicotinamide adenine dinucleotide phosphate hydrate $(\beta$-NADP), D-glucose 6-phosphate sodium salt (G-6-P), and glucose-6-phosphate dehydrogenase (G-6-P-DE) used in the present study were purchased from Sigma-Aldrich (St. Louis, MO, United States). Heparin was purchased from HuiShi Biochemical Reagent Co., Ltd. (Shanghai, China). De-ionized water was prepared by a Milli-Q system (Millipore, Burlington, MA, United States). Acetonitrile, methanol, and formic acid (Sigma-Aldrich) were of HPLC grade. Other reagents were of analytical grade.

\section{Preparation of the Water Decoction of Datura metel Seeds (WDS)}

Datura metel seeds were collected from the medicinal botanical garden of Heilongjiang University of Traditional Chinese Medicine. The authenticity of the sample was identified by Dr. Ruifeng Fan, Botanist, Department of Medicinal Plant, Heilongjiang University of Traditional Chinese Medicine. The voucher specimen (specimen number: 2016035) were preserved in the laboratory of Chinese Medicine Chemistry, Heilongjiang University of Traditional Chinese Medicine. The WDS was prepared by extracting $100 \mathrm{~g}$ of $\mathrm{D}$. metel seeds decocted in $600 \mathrm{~mL}$ water three times $(2.0,2.0,1.0 \mathrm{~h})$. The decoctions were combined, filtered, and concentrated in vacuum at $50^{\circ} \mathrm{C}$. The final concentration of the extract was $0.01 \mathrm{~g} / \mathrm{mL}$ (crude drug per $\mathrm{g} / \mathrm{mL}$ ).

\section{Animals and Drug Administration}

Male Sprague-Dawley rats (180-220 g) were purchased from the Laboratory Animal Center of the Second Affiliated Hospital of Harbin Medical University (Heilongjiang Province, China). The rats were kept in metabolic cages $(465 \mathrm{~mm} \times 300 \mathrm{~mm} \times 200 \mathrm{~mm})$, and the breeding room was at $25^{\circ} \mathrm{C}$ and $60 \pm 5 \%$ relative humidity. All animals had free access to water and normal chow was provided ad libitum at a $12 \mathrm{~h}$ dark-light cycle for 3 days, and then fasted for $12 \mathrm{~h}$ before the experiments. The animal facilities and protocols were approved by the Animal Care and Use Committee of the Harbin Medical University. All procedures were in accordance with the National Institutes of Health Guide for the Care and Use of Laboratory Animals (ILAR, 1996).

The pure compounds, including 1, 3, 5, 9, 10, 13, 18, 20, 22, 29, 32, 40, and 41, were suspended in $1 \%$ carboxy-methyl cellulose-sodium and were separately provided to rats $(n=4)$ orally at $20 \mathrm{mg} / \mathrm{kg}$. The WDS was provided to rats $(n=4)$ at $0.5 \mathrm{~g} / \mathrm{kg}$ (clinical dosage, equivalent to $600 \mathrm{mg} /$ day for a $60-\mathrm{kg}$ human), respectively. The dosage in our study was the clinically safe and effective (Pharmacopoeia Commission, 2015). The control group $(n=4)$ was administrated with $1000 \mu \mathrm{L}$ normal saline. 

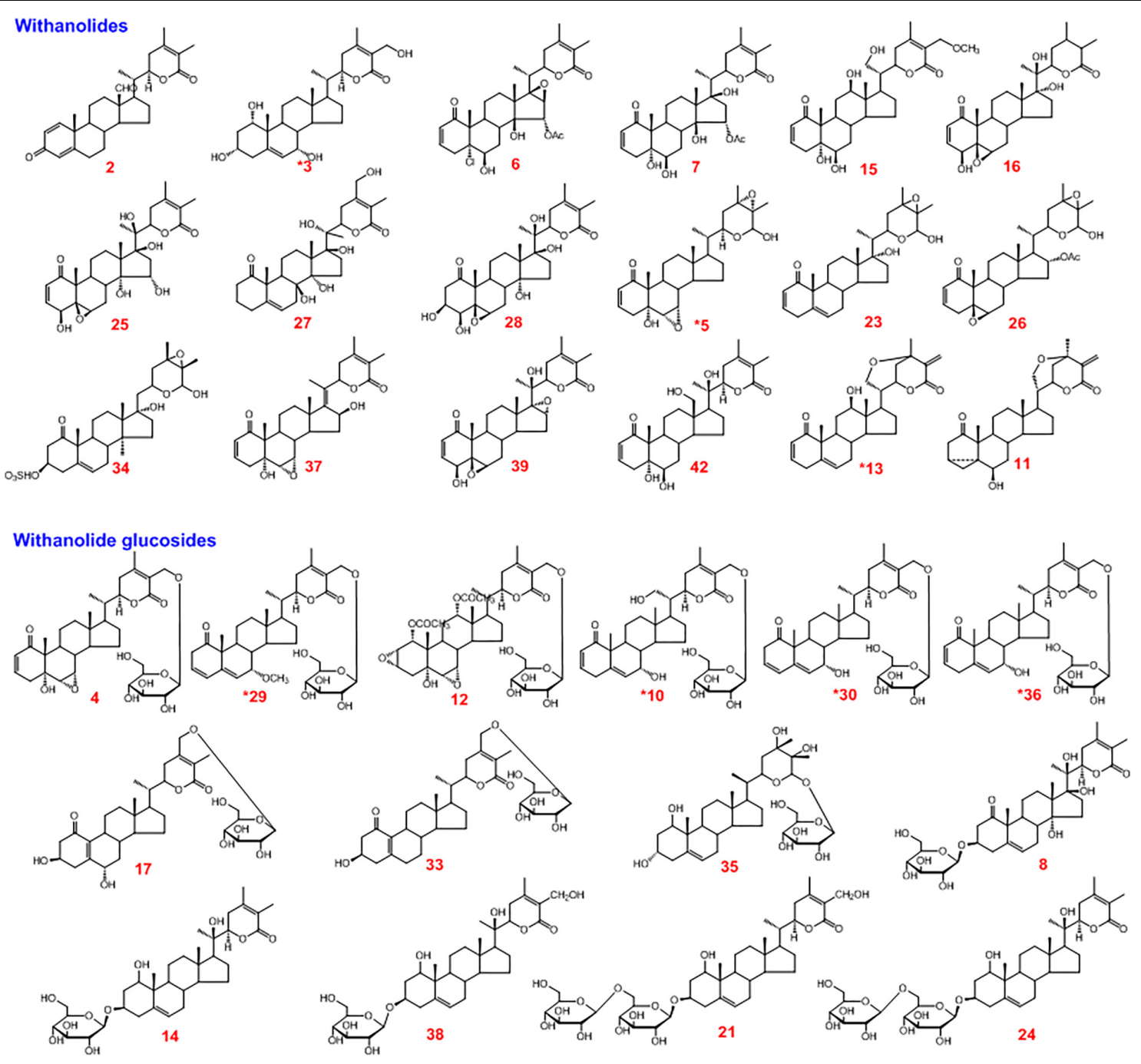

Amides
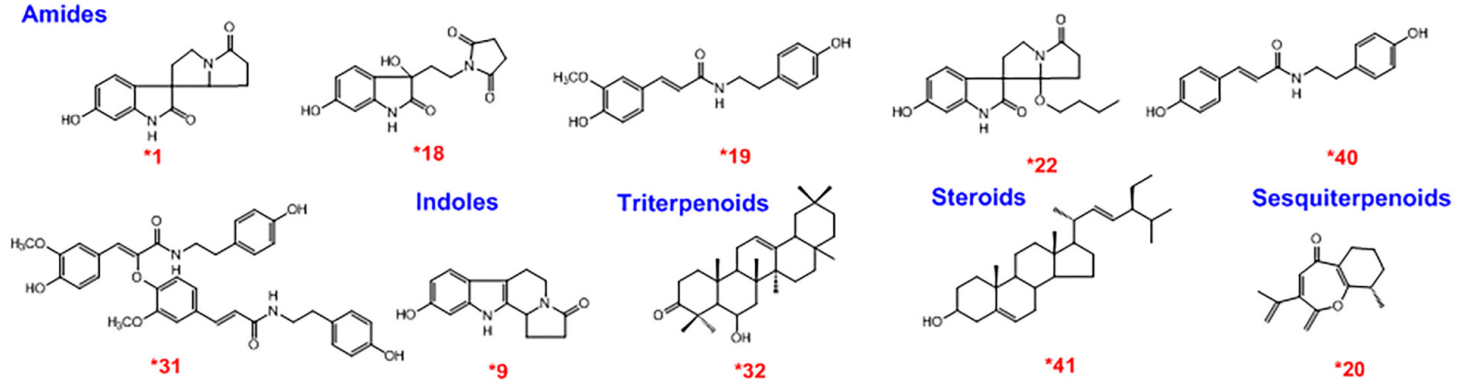

FIGURE 2 | Structure of the chemical constituents of the water extract of Datura metel seeds (WDS).*Identified by comparing with reference standards.

\section{Preparation of Plasma, Urine, and Fecal Samples}

To obtain plasma samples, blood $(1000 \mu \mathrm{L})$ was collected from the angular vein into heparinized tubes at six time points: 0.5 , $1,2,4,6$, and $10 \mathrm{~h}$ after the administration of WDS and pure compounds $(n=4)$. The blood of two rats was collected at $0.5,2$, and $6 \mathrm{~h}$, or at 1,4 , and $10 \mathrm{~h}$, and then centrifuged at $10,000 \times g$ for $10 \mathrm{~min}$ at $4^{\circ} \mathrm{C}$ to obtain the plasma and then pooled plasma samples of different time points. Urine and fecal samples were collected for $0-24 \mathrm{~h}$ from rats held in metabolic cages (DXL-D, Keke Medical Model Co., Ltd., Shanghai, China).

Plasma, urine, and fecal samples were prepared as described in our previous report (Xu et al., 2018). The residue of these samples was dissolved in $300 \mu \mathrm{L}$ of methanol, and filtered 
through a $0.22-\mu \mathrm{m}$ membrane for ultra-high performance liquid chromatography coupled with electrospray ionization (UPLC/ESI) qTOF-MS analysis.

\section{Incubation of Rat Liver Microsomes}

Compounds 3, 5, 10, 13, 29, 40, and 41 were separately dissolved in methanol, and then diluted with phosphate buffered saline (PBS). The final concentration of each compound in the $200-\mu \mathrm{L}$ incubation mixture (NADPH-generating system, $100 \mathrm{mM}$ potassium phosphate buffer ( $\mathrm{pH}$ 7.4), and rat liver microsomes) was $25 \mu \mathrm{M}$, and the amount of organic solvent in the mixture was lower than $1 \%(\mathrm{v} / \mathrm{v})$. PBS-containing methanol was added as the negative control. The incubation was conducted at $37^{\circ} \mathrm{C}$ for $2 \mathrm{~h}$. After this period, the reaction was terminated by adding $1000 \mu \mathrm{L}$ of cold acetonitrile. The mixture was then kept at $4^{\circ} \mathrm{C}$ for $30 \mathrm{~min}$, and the precipitated protein was removed by centrifugation at $10,000 \times \mathrm{g}$ for $10 \mathrm{~min}$ at $4^{\circ} \mathrm{C}$.

\section{Enzyme Hydrolysis}

A $100-\mu \mathrm{L}$ aliquot of plasma or urine sample was dried under a gentle flow of nitrogen gas and then mixed with $400 \mu \mathrm{L}$ of b-glucuronidase solution (containing $19.86 \mathrm{U} / \mu \mathrm{L}$, in sodium acetate buffer, $\mathrm{pH}$ 5.5). The mixture was vortexed for $5 \mathrm{~min}$, incubated in a $37^{\circ} \mathrm{C}$ water bath for $1.5 \mathrm{~h}$, treated with $1000 \mu \mathrm{L}$ of cold methanol-acetonitrile $(1: 1, \mathrm{v} / \mathrm{v})$ to precipitate the protein, and then centrifuged at $10,000 \times g$ for $10 \mathrm{~min}$ at $4^{\circ} \mathrm{C}$. The supernatant was dried under a gentle nitrogen flow and then dissolved in $100 \mu \mathrm{L}$ of methanol. The solution was filtered through a $0.22-\mu \mathrm{m}$ membrane for analysis.

\section{UPLC/ESI/qTOF-MS Analysis}

The analysis was performed on an ACQUITY UPLC instrument coupled with a Xevo G2 qTOF mass spectrometer (Waters, Milford, MA, United States) via an ESI ion source. The autosampler was controlled via the MassLynx ${ }^{\mathrm{TM}} 4.1$ software (Waters). Samples were separated on an ACQUITY UPLC HSS T3 column $(2.1 \mathrm{~mm} \times 100 \mathrm{~mm}, 1.7 \mu \mathrm{m}$; Waters $)$. The mobile phase consisted of acetonitrile (A) and water containing $0.1 \%(\mathrm{v} / \mathrm{v})$ formic acid (B) at a flow rate of $400 \mu \mathrm{L} / \mathrm{min}$. The gradient elution program was set as follows: 0-7 $\mathrm{min}, 10-22 \% \mathrm{~A}$; 7-13 $\mathrm{min}, 22-70 \%$ A; 13-17 $\mathrm{min}, 70-90 \%$ A; 17-20 $\mathrm{min}, 90-95 \%$
A; 20-22 $\min , 95 \%$ A. A 2- $\mu \mathrm{L}$ sample aliquot was injected for analysis, and the column temperature was $40^{\circ} \mathrm{C}$.

The qTOF/MS system was equipped with an ESI source operating in positive ion mode, as in our previous report ( $\mathrm{Xu}$ et al., 2018). High-purity nitrogen $\left(\mathrm{N}_{2}\right)$ and high-purity helium (He) were used as desolvation gas and collision gas, respectively. The flow rate of $\mathrm{N}_{2}$ was $600 \mathrm{~L} / \mathrm{h}$ and that of He was $50 \mathrm{~L} / \mathrm{h}$. The optimized parameters were: capillary voltage, $3.5 \mathrm{kV}$; sample cone voltage, $30 \mathrm{~V}$; and extraction cone voltage, $4 \mathrm{~V}$. The desolvation and source temperatures were $350^{\circ} \mathrm{C}$ and $100^{\circ} \mathrm{C}$, respectively. The MS full scan range was $100-1000 \mathrm{~m} / z$, and $\mathrm{MS}^{\mathrm{n}}$ range was $100-800 \mathrm{~m} / z$. All data collected in the positive ion mode were acquired and processed by the MassLynx ${ }^{\text {TM }}$ 4.1 software (Waters). UHPLC/qTOF-MS is the high-resolution mass spectra, which could provide the accurate $[\mathrm{M}+\mathrm{H}]^{+}$ions of the metabolites.

\section{Quadrupole Ion Trap (Q TRAP) LC/MS/MS}

The AB SCIEX 4000 Q TRAP ${ }^{\mathrm{TM}}$ composite triple quadrupole/linear ion trap tandem mass spectrometer (SCIEX, Framingham, MA, United States) connected to the UHPLC via the ESI interface (Applied Biosystems, Foster City, CA, United States) was operated in the positive ion mode. The column effluent was split using a zerodead-volume " $\mathrm{T}$ " connector, with approximately half of the flow being fed to the mass spectrometer. The interface and parameters of the mass spectrometer were as follows: spray capillary voltage, $5.5 \mathrm{kV}$; DP, $70 \mathrm{~V}$; EP, $0 \mathrm{~V}$; CE, $45 \mathrm{~V}$; nebulizer pressure, 40 psi; dry gas pressure, 40 psi; curtain gas pressure, 10 psi; and dry gas temperature, $600^{\circ} \mathrm{C}$. All data were acquired and processed using Analyst (SCIEX). The mass spectrum fragment ions of $\mathrm{MS}^{2}$ and $\mathrm{MS}^{3}$ could be obtained by Q-TRAP LC/MS/MS.

\section{RESULTS AND DISCUSSION}

\section{Characterization of the Chemical Constituents of WDS}

A rapid and sensitive UHPLC/qTOF-MS method was employed to characterize the chemical constituents of WDS. Forty-two compounds (1-42) were identified or tentatively characterized

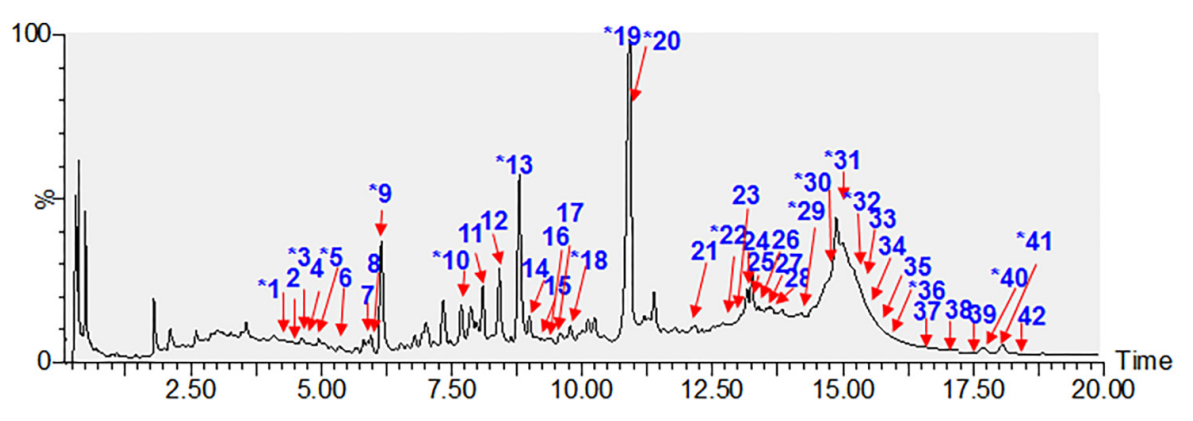

FIGURE 3 | LC/MS total ion currents of the water extract of Datura metel seeds (WDS). *Identified by comparing with reference standards. 


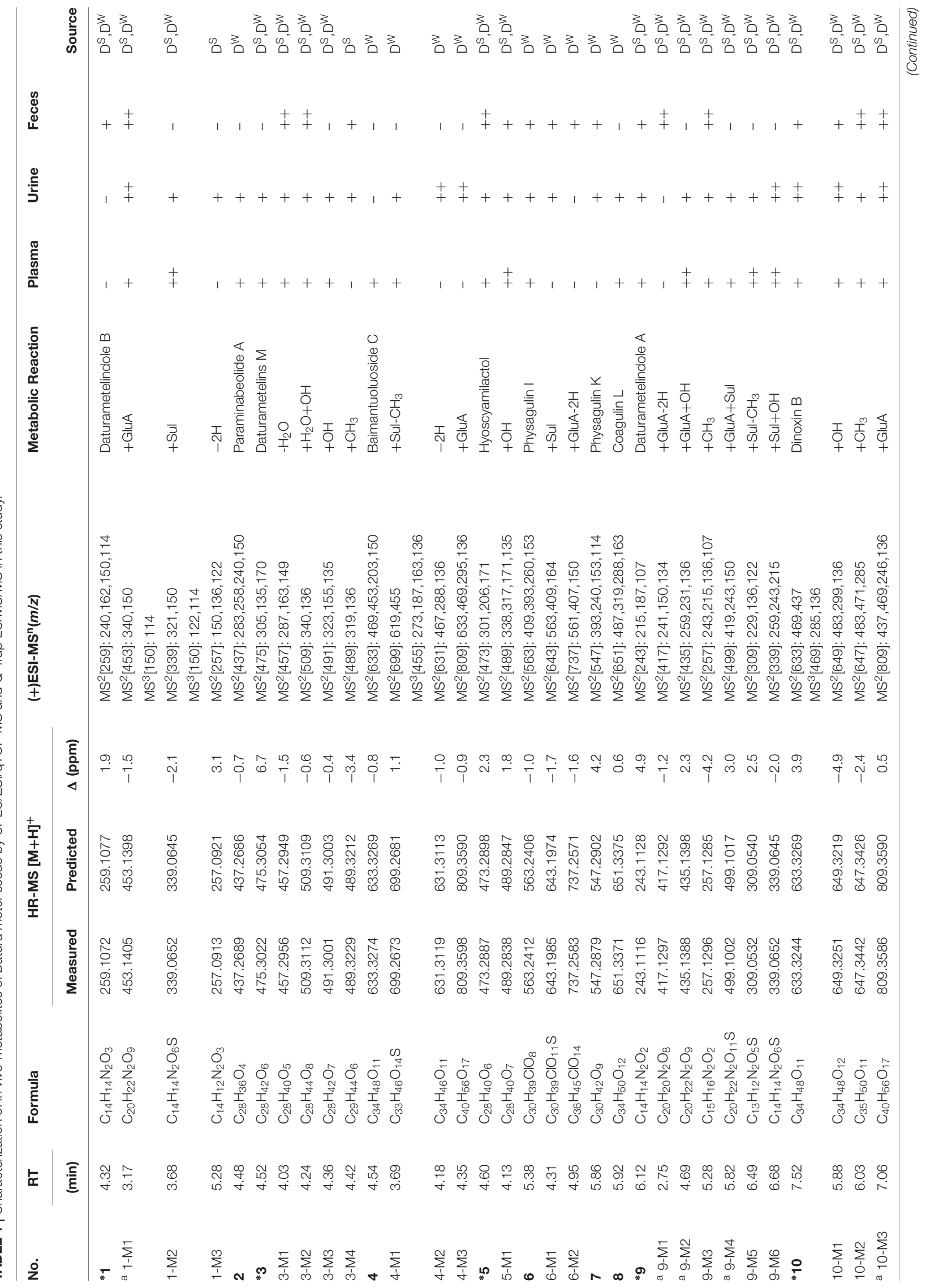




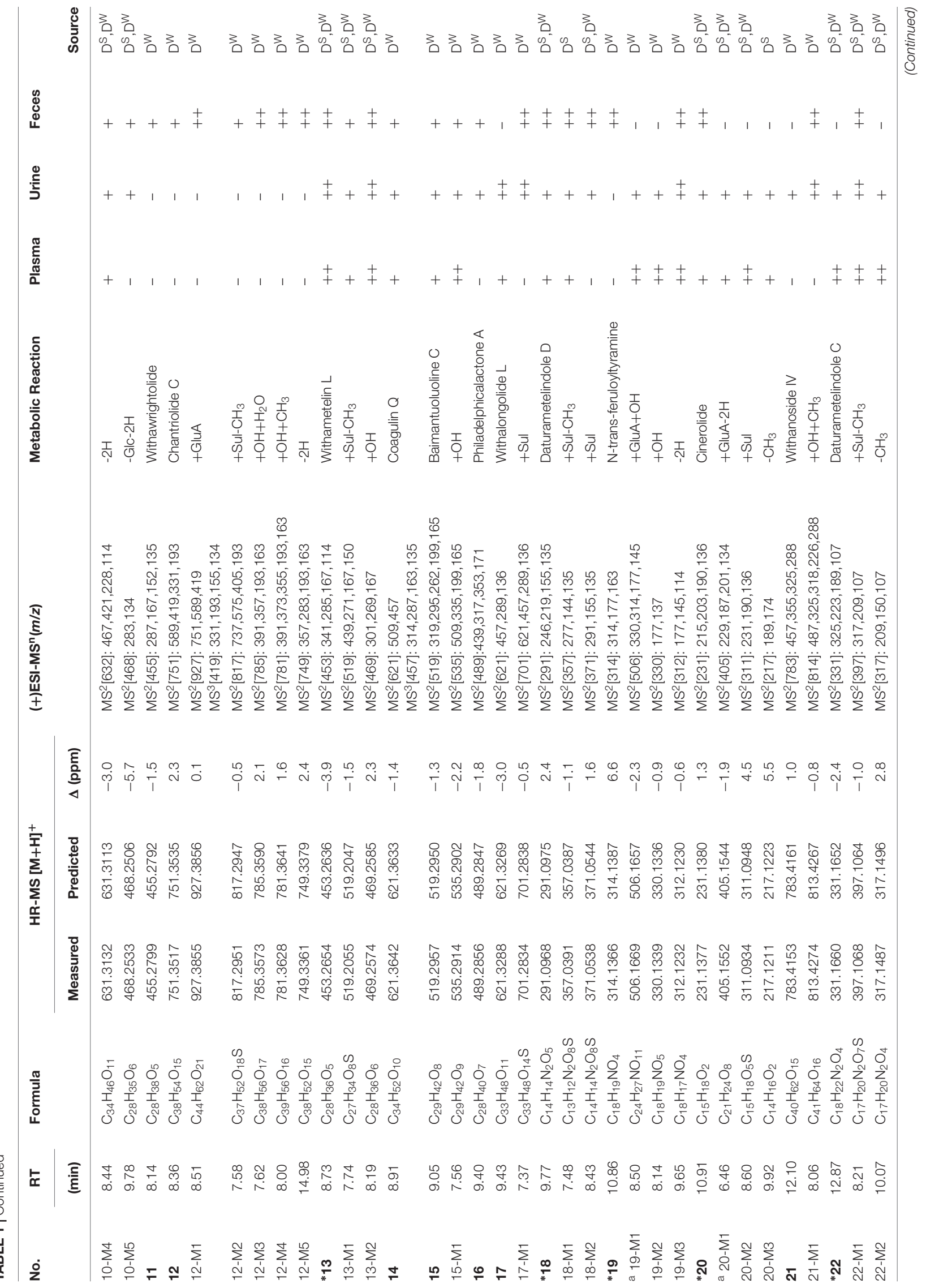




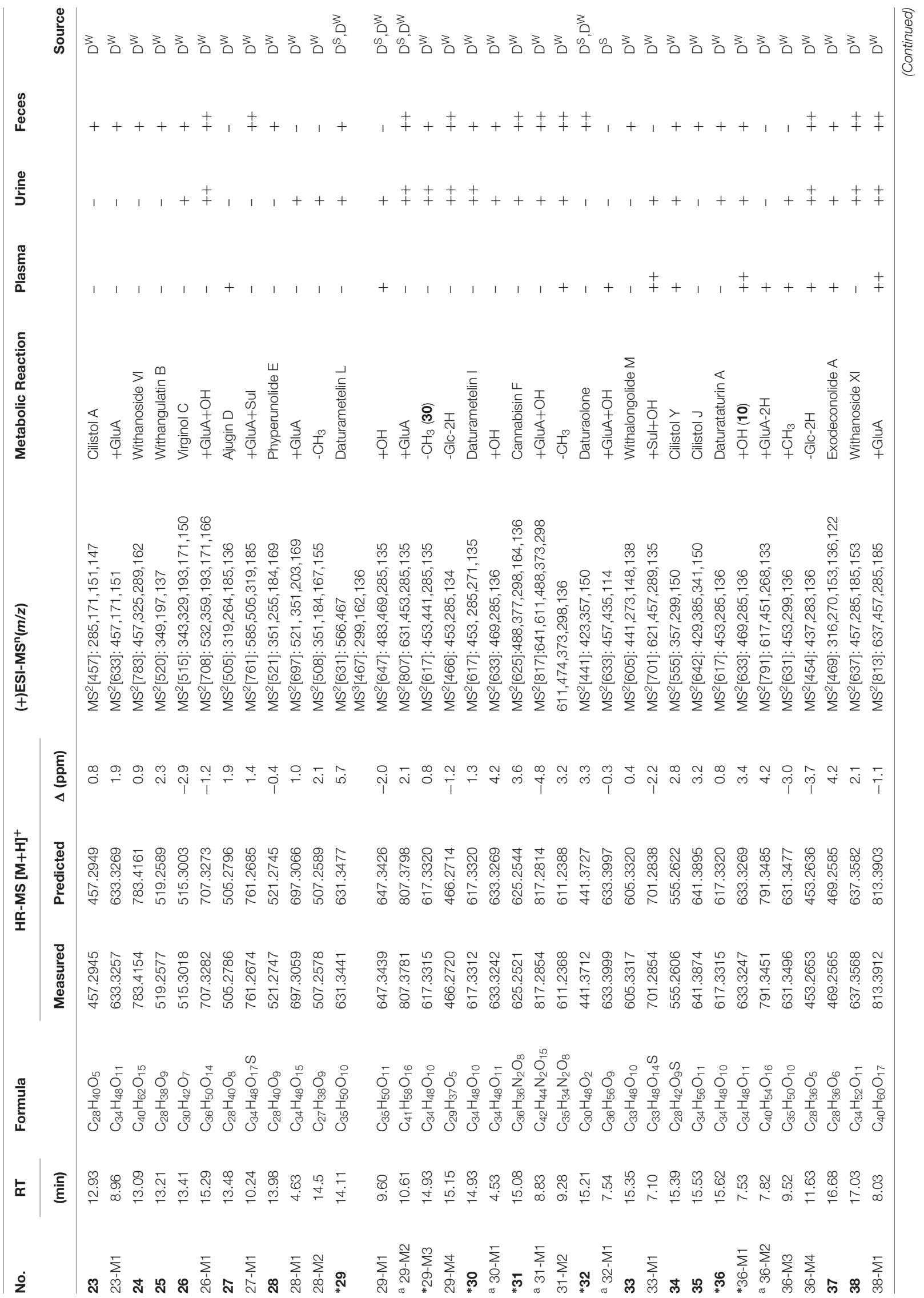




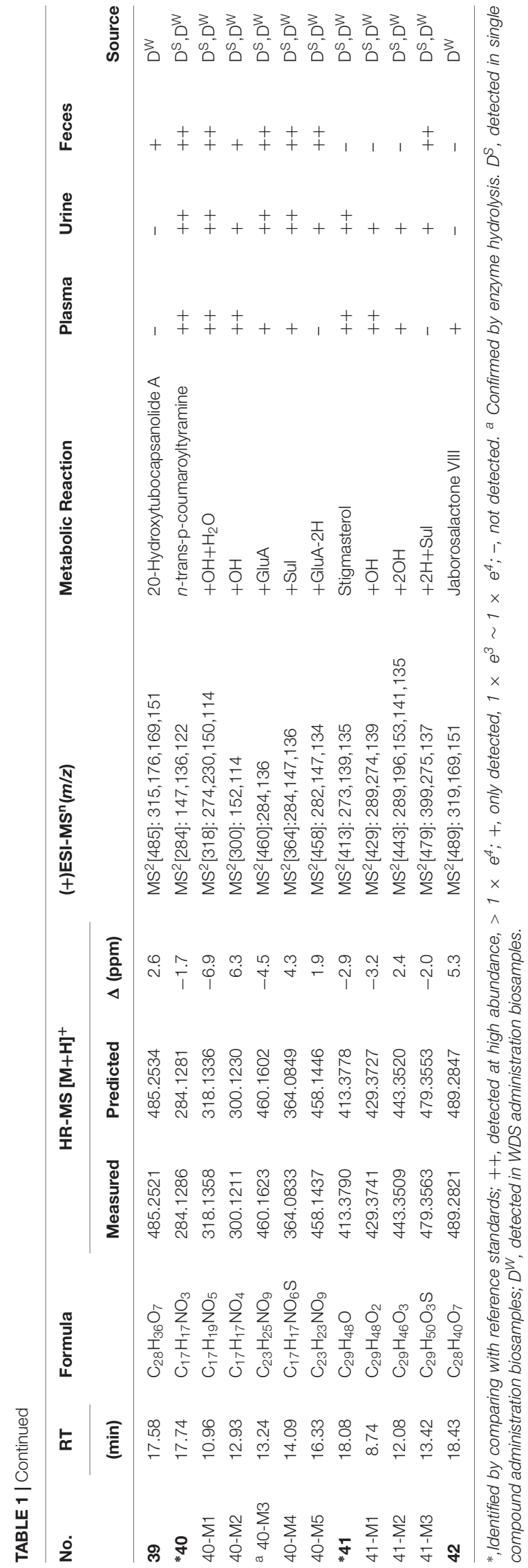

(Figure 3), including 18 withanolides, 14 withanolide glucosides, six amides, one indole, one triterpenoid, one steroid, and one sesquiterpenoid. Among them, 17 compounds (1, 3, 5, $9,10,13,18,19,20,22,29,30,31,32,36,40$, and 41) were unambiguously identified by a comparison with reference standards. Another 25 potential constituents $(\mathbf{2}, \mathbf{4}, \mathbf{6}, \mathbf{7}, \mathbf{8}, \mathbf{1 1}$, $12,14,15,16,17,21,23,24,25,26,27,28,33,34,35,37$, 38,39 , and 42 ) were tentatively characterized based on their retention times, UV spectra, high-accuracy mass spectra, and MS/MS fragmentation behaviors. Chemical structures of the single compounds characterized in WDS are given in Figure 2.

\section{Metabolic Pathways of Single Representative Compounds}

The chemical constituents of WDS were classified into seven groups according to their structural type: withanolides (A), withanolide glucosides (B), amides (C), indoles (D), triterpenoids $(\mathrm{E})$, steroids $(\mathrm{F})$, and sesquiterpenoids $(\mathrm{G})$. In our previous study, we selected four representative compounds containing two withanolide glucosides (daturataturin A and daturametelin $\mathrm{I}$ ), and two amides (cannabisin $\mathrm{F}$ and $n$-trans-feruloyltyramine) from WDS and elucidated their metabolic pathways (Xu et al., 2018). In the present study, we used these pathways for metabolite identification of other compounds, which had similar scaffolds in WDS, developing a "compound to extract" strategy. The metabolic pathways of daturametelindole $B(1)$, daturametelin $M$ (3), hyoscyamilactol (5), daturametelindole A (9), dinoxin B (10), withametelin L (13), daturametelindole D (18), cinerolide (20), daturametelindole C (22), daturametelin L (29), daturaolone (32), $n$-trans-p-coumaroyltyramine (40), and stigmasterol (41) were first reported in this study, and these 13 single compounds were selected to represent seven major scaffolds in WDS. These compounds were labeled in the HPLC fingerprint of WDS. As shown in Table 1, the multistage mass spectrum fragmentation information (ESI-MS ${ }^{\mathrm{n}}$ ) was obtained both from UPLC/ESI/qTOF-MS and Q TRAP LC/MS/MS analysis.

\section{Withanolides}

Compounds 3, 5, and 13 were chosen as representatives of withanolides (Figure 2). They were considered as bioactive constituents (Ma et al., 1999; Zhang et al., 2018) and could be detected per se in plasma and urine samples. Hydroxylation was the major metabolic reaction for withanolides from $D$. metel (Table 1). In addition, compounds $\mathbf{3}$ and $\mathbf{5}$ may be metabolized into 21- $\mathrm{OH}$ daturametelin $\mathrm{M}$ and 21- $\mathrm{OH}$ hyoscyamilactol, respectively. This reaction was also observed for daturataturin $\mathrm{A}$ (36) and daturametelin I (30) in our previous study (Xu et al., 2018). However, the hydroxyl position of $\mathbf{1 3}$ could not be assigned due to the limited structural information. Hydroxylated products were also detected when $\mathbf{3}, \mathbf{5}$, and $\mathbf{1 3}$ were incubated in rat liver microsomes, indicating that the hydroxylation reaction was catalyzed by P450 enzymes (Supplementary Figure S1).

\section{Withanolide Glucosides}

The metabolism of two withanolide glucosides, $\mathbf{1 0}$ and 29, was investigated. When $O$-glycosides lose the sugar residue to produce corresponding aglycones (withanolides), 

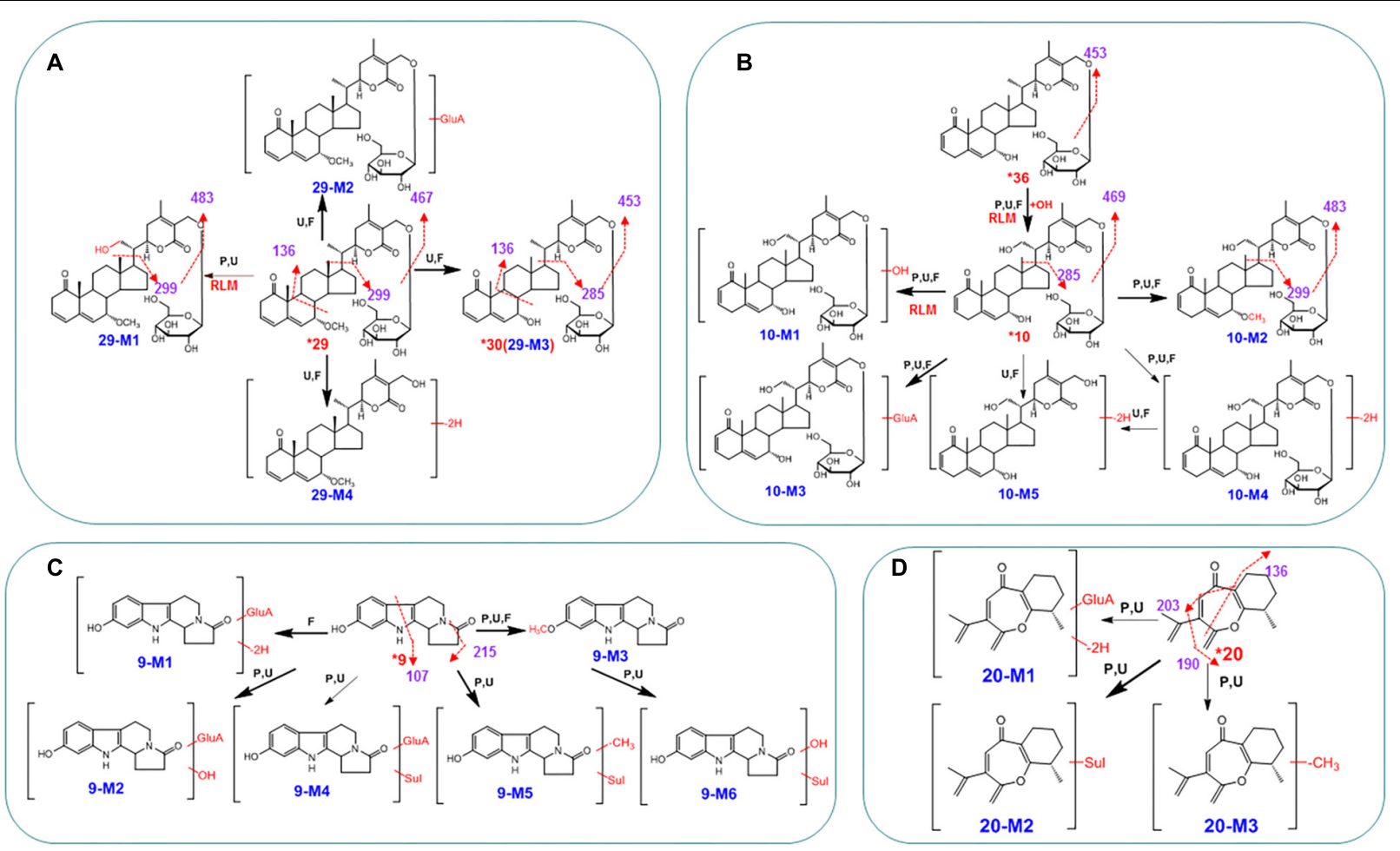

FIGURE 4 | The proposed metabolic pathways for compounds $\mathbf{9}$ (C), 10 (B), 20 (D), and $\mathbf{2 9}$ (A) in rats after the oral administration of the WDS. Red bold arrows indicate major metabolites; *, compared with reference standards; U, detected in urine samples; P, detected in plasma samples; F, detected in fecal samples; RLM, detected in rat liver microsomes; Sul, sulfate; GluA, glucuronic acid residue.

dehydrogenation occurs. This reaction of interconversion was very common for withanolide glucosides. In addition, hydroxylation, (de)methylation, and glucuronidation of withanolide glucosides were also major reactions as shown in Figures 4A,B. These metabolites, derived from the three withanolide glucosides, were detected in plasma, urine, and fecal samples (Table 1). For example, 29 produced the hydroxylated metabolite 29-M1 $(\mathrm{m} / \mathrm{z}$ 647 $\rightarrow$ 483, 469, 299, 136), the demethylated metabolite 29-M3 $(\mathrm{m} / z$ 617 $\rightarrow 453,285$, 136, identified as 30), the glucuronide-conjugated metabolite 29-M2 $(\mathrm{m} / \mathrm{z} 807 \rightarrow 631,453,285,136)$, and hydrolyzed and dehydrogenated metabolite 29-M4 $(\mathrm{m} / z$ 466 $\rightarrow 453,285,134)$.

\section{Amides}

Amides are abundant in D. metel seeds, and considered as characteristic components (Karim et al., 2017). Four major amides $(\mathbf{1}, \mathbf{1 8}, \mathbf{2 2}$, and 40) were chosen to examine the in vivo metabolism of this type of compounds. In brief, the metabolism of amides varied significantly according to the group substitutions on their backbones. For example, 1 and 40 could undertake different phase I reactions, including hydroxylation, dehydrogenation, hydroxylation, and hydration. Phase II conjugation reactions (to form glucuronides and sulfates) were common in amides (Table 1), but the peaks of glucuronides disappeared when the sample was treated with $\beta$-glucuronidase (Supplementary Figure S2). Amides 18 and 22 were mainly involved in demethylation and sulfation. The major metabolic reactions and metabolite distributions of these four amides are listed in Table 1. Plasma and urine samples mainly contained phase II metabolites and hydroxylated products, while urine samples comprised most metabolites.

\section{Indoles}

Indole alkaloids in D. metel seeds are extremely important, and their distribution and metabolism have been reported in several different organisms (Gillam et al., 2000; van der Fits and Memelink, 2000; Rischer et al., 2006; Ziegler and Facchini, 2008). We chose daturametelindole A (9) for examining metabolic pathways. The qTOF mass spectra showed $[\mathrm{M}+\mathrm{H}]^{+}$ ions at $\mathrm{m} / \mathrm{z} 243.1116$, consistent with the molecular formula of $\mathrm{C}_{14} \mathrm{H}_{14} \mathrm{~N}_{2} \mathrm{O}_{2}$. In the ion trap $\mathrm{MS}^{\mathrm{n}}$ spectra, the $[\mathrm{M}+\mathrm{H}]^{+}$ ions could further add a glucuronic acid moiety (176 U), and then be dehydrogenized, hydroxylated, and sulfated to produce the corresponding ions at $m / z 417$ (9-M1), 435 (9-M2), and 499 (9-M4), respectively (Figure 4C). The above conjugates were confirmed by enzyme hydrolysis. When indole 9 from the plasma sample was treated with $\beta$-glucuronidase, the peaks of 9-M1, 9-M2, and 9-M4 disappeared, and the peak corresponding to 9 increased remarkably (Figure 4C). Thus, it could be deduced that these metabolites were glucuronides of 9. The sulfate conjugates 9-M5 and 9-M6 were detected in plasma, urine, and fecal samples. Their MS/MS spectra were dominated by the neutral loss of $80 \mathrm{Da}$. Metabolite 9-M3 was highly abundant in fecal samples. Its high-resolution mass spectra 


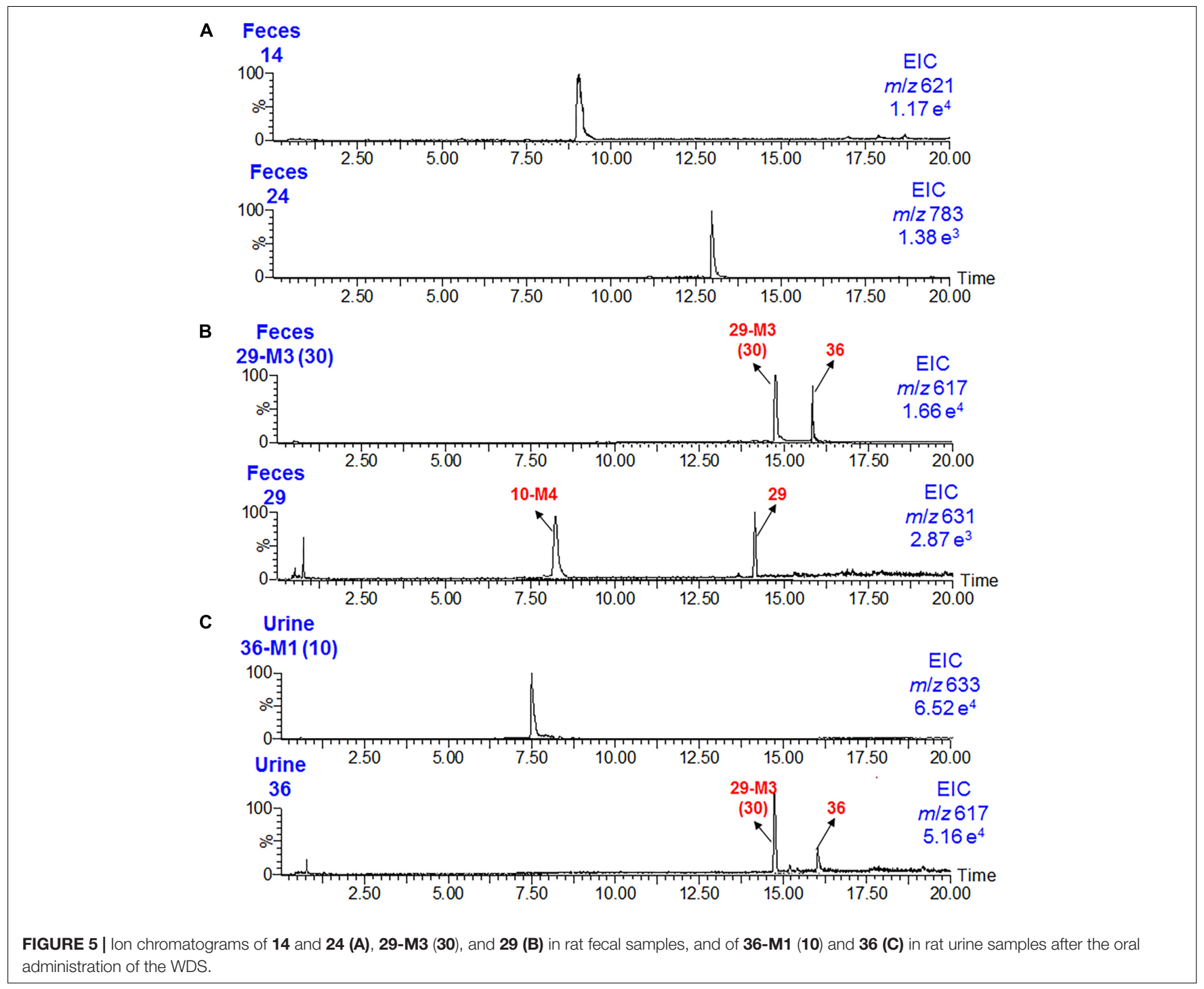

showed an $[\mathrm{M}+\mathrm{H}]^{+}$ion at $m / z 257.1296$, corresponding to the molecular formula $\mathrm{C}_{15} \mathrm{H}_{16} \mathrm{~N}_{2} \mathrm{O}_{2}$. In the tandem mass spectra, 9-M3 produced fragment ions at $\mathrm{m} / z 243\left(\left[\mathrm{M}+\mathrm{H}-\mathrm{CH}_{3}\right]^{+}\right)$, $\mathrm{m} / z 215\left(\left[\mathrm{M}+\mathrm{H}-\mathrm{CH}_{3} \cdot-\mathrm{CO}\right]^{+}\right)$, and $\mathrm{m} / z 107\left(\left[\mathrm{M}+\mathrm{H}-\mathrm{CH}_{3}-\mathrm{CO}-\right.\right.$ $\left.\mathrm{C}_{7} \mathrm{H}_{9} \mathrm{~N}\right]^{+}$), indicating that it was methylated metabolite. The metabolic pathways of 9 are illustrated in Figure 4C.

\section{Triterpenoids, Steroids, and Sesquiterpenoids}

In addition to the major structural types (A) to (D), less abundant groups of compounds were also found in WDS in previous chemical studies (Kuang et al., 2009; Yang et al., 2014; Bhardwaj et al., 2016). They were taken into consideration in this study, and the representative scaffolds included triterpenoids (32, daturaolone), steroids (41, stigmasterol), and sesquiterpenoids (20, cinerolide). Identification of 32, 41, and $\mathbf{2 0}$ is depicted in Table $\mathbf{1 .}$

When 32 was provided to rats, high amounts of prototype were detected in fecal samples. The methoxyl groups at C-23, -14, -29 , or -30 may undergo hydroxylation, followed by glucuronide conjugation (Table 1; Sanchez-Gonzalez et al., 2015). The phase I and phase II metabolites of $\mathbf{4 1}$ were also observed in vivo samples. Among phase I metabolites, the hydroxylation products were extensively observed. This type of reaction involved the addition of one or two hydroxyl groups to the parent drug. The phase II biotransformation was mainly sulfation, and the phase II metabolite 41-M3 was formed by a reduction reaction followed by a sulfated reaction. Most steroids were metabolized in the same way as ganoderic acid D (Cheng et al., 2012). After $20 \mathrm{mg} / \mathrm{kg}$ oral administration, a large portion of $\mathbf{2 0}$ was metabolized. Although this compound was not observed in plasma samples, it occurred in the unchanged form in fecal samples. The UPLC/ESI/qTOF-MS analysis detected large proportions of three metabolites in plasma samples (Table 1). The high-resolution mass spectra of $\mathbf{2 0 -} \mathbf{M} 3$ revealed a $[\mathrm{M}+\mathrm{H}]^{+}$ signal at $\mathrm{m} / \mathrm{z} 217.1211$, indicating the molecular formula $\mathrm{C}_{14} \mathrm{H}_{16} \mathrm{O}_{2}$, corresponding to a demethylated derivative of $\mathbf{2 0}$. Similar to cyperon (Duan et al., 2012), 20 presented fragment ions at $\mathrm{m} / z 231\left([\mathrm{M}+\mathrm{H}-\mathrm{O}]^{+}\right), 203\left([\mathrm{M}+\mathrm{H}-\mathrm{CO}]^{+}\right)$, and 190 
$\left(\left[\mathrm{M}+\mathrm{H}-\mathrm{C}_{3} \mathrm{H}_{5}\right]^{+}\right)$(Figure 4D). Two phase II metabolites of 20 were detected, namely, glucuronide conjugate (20-M1) and sulfate conjugate (20-M2) (Figure 4D), which disappeared when the sample was treated with $\beta$-glucuronidase.

\section{Characterization of WDS Metabolites at a Clinical Dosage}

To mimic traditional Chinese medicine clinical use, D. metel seeds were cooked in water to obtain the WDS extract. The dosage of WDS provided to rats $(0.15 \mathrm{~g} / \mathrm{kg})$ was equivalent to the $1.5 \mathrm{~g} /$ day for a $60-\mathrm{kg}$ human (Pharmacopoeia Commission, 2015). Under these conditions, 61 metabolites (including intact original compounds) were detected in rat plasma samples, 83 in urine samples, and 76 in fecal samples using qTOF-MS and LC/MS/MS. Withanolides, withanolide glucosides, and alkaloids were the major absorbed chemical components of WDS, and their oral bioavailability was as follows: withanolides $>$ withanolide glucosides $>$ amides and indoles. The contents of compounds $\mathbf{9}$, 13, and 20 are very high in WDS, and they could also be easily detected in WDS-administrated plasma samples. Interestingly, as shown in Table 1 and Figure 5, withanolide monoglucoside (14) appeared to have a better oral bioavailability than withanolide diglucoside (24). In addition, hydroxylation or (de)methylation was the most common reaction for withanolide glucosides. The absorption of the demethylated metabolite occurred at 7-OH (for instance 29-M3, 30) was easier than the prototypes (29); besides that, the hydroxylated metabolite at C-21 (36-M1, 10) could be readily absorbed into circulation than compound per se (36) (Figures 4, 5).

Overall, and according to the metabolic pathways of single representative compounds, the major reactions of the WDS extract included phase II metabolism, hydroxylation, (de)methylation, and dehydrogenation. The phase II metabolites were confirmed by $\beta$-glucuronidase hydrolysis. The hydroxylation of $3,5,10,13,29,40$, and 41 was confirmed by rat liver microsomes incubation experiments, indicating that this hydroxylation was catalyzed by P450 enzymes. Withanolides and withanolide glucosides without a phenolic hydroxyl group at C-21 might readily undergo monohydroxylation. Compounds 9, 10, and 36 could add a methyl group, and compounds 20, 21, 28, and 31 could lose a methyl group. Finally, five dehydrogenated metabolites (1-M5, 4-M2, 10-M4, 12-M5, and 19-M3) were characterized by LC/MS analysis (Table 1). Among the metabolic reactions described above, the phase II metabolism (glucuronidation or sulfation) was observed most commonly in the metabolism of WDS constituents.

\section{REFERENCES}

Bachheti, R. K., Rai, I., Mishra, V. K., and Joshi, A. (2018). Antioxidant and antimicrobial properties of seed oil of Datura metel. J. Environ. Biol. 39, 182-188. doi: 10.22438/jeb/39/2/mrn-341

Bellila, A., Tremblay, C., Pichette, A., Marzouk, B., Mshvildadze, V., Lavoie, S., et al. (2011). Cytotoxic activity of withanolides isolated from Tunisian Datura metel L. Phytochemistry 72, 2031-2036. doi: 10.1016/j.phytochem.2011.07.009

Bhardwaj, K., Kumar, S., and Ojha, S. (2016). Antioxidant activity and FT-IR analysis of Datura innoxia and Datura metel leaf and seed

\section{CONCLUSION}

We propose the strategy "chemical analysis, metabolism of single representative compounds, and metabolism of the extract at the clinical dosage" to systematically characterize the in vivo metabolites of $D$. metel seeds. After the oral administration of $D$. metel seeds water extract to rats at a normal clinical dosage $(0.15 \mathrm{~g} / \mathrm{kg}), 113$ phytochemicals (including intact original compounds) were identified or tentatively characterized by a highly sensitive LC/MS-MS method. As far as we know, this is the first report on the full metabolic profiling of the D. metel seeds water extract in vivo. This strategy could be helpful for pharmacokinetic studies of multi-component herbal products.

\section{ETHICS STATEMENT}

The animal facilities and protocols were approved by the Animal Care and Use Committee of Harbin Medical University. All procedures were in accordance with the National Institutes of Health Guide for the Care and Use of Laboratory Animals (ILAR, 1996).

\section{AUTHOR CONTRIBUTIONS}

QW, BY, and CX participated in research design. CX, YL, HQ, $\mathrm{LN}, \mathrm{YZ}, \mathrm{WL}, \mathrm{XX}$, and YS conducted the experiments. QW, CX, and YL performed the data analysis.

\section{FUNDING}

This work was supported by China Postdoctoral Science Fund (Grant No. 2018M630377), Fundamental Research Funds for the Provincial Universities (Grant No. 2017JCZX59), and Doctoral Fund of The 2nd Affiliated Hospital of Harbin Medical University (Grant No. BS2011-19).

\section{SUPPLEMENTARY MATERIAL}

The Supplementary Material for this article can be found online at: https://www.frontiersin.org/articles/10.3389/fphar. 2019.00571/full\#supplementary-material

methanolic extracts. Afr. J. Tradit. Complem. 13, 7-16. doi: 10.21010/ajtcam. v13i5.2

Cheng, C. R., Yang, M., Yu, K., Guan, S. H., Tao, S. J., Millar, A., et al. (2012). Identification of metabolites of ganoderic acid $\mathrm{D}$ by ultra-performance liquid chromatography/quadrupole time-of-flight mass spectrometry. Drug Metab. Dispos. 40, 2307-2314. doi: 10.1124/dmd.112.047506

Duan, S. N., Qi, W., Zhang, S. W., Huang, K. K., and Yuan, D. (2012). Ultra high performance liquid chromatography coupled with electrospray ionization/quadrupole time-of-flight mass spectrometry for the rapid analysis of constituents in the traditional Chinese 
medicine formula Wu Ji Bai Feng Pill. J. Sep. Sci. 40, 3977-3986. doi: $10.1002 /$ jssc. 201700438

Gillam, E. M. J., Notley, L. M., Cai, H. L., De Voss, J. J., and Guengerich, F. P. (2000). Oxidation of indole by cytochrome P450 enzymes. Biochemistry 39, 13817-13824. doi: 10.1021/bi001229u

Gu, M., Yu, Y., Gumaherath, G., Gunatilaka, A., Li, D., and Sun, D. (2014). Structure-activity relationship (SAR) of withanolides to inhibit Hsp90 for its activity in pancreatic cancer cells. Invest. New Drugs 32, 68-74. doi: 10.1007/ s10637-013-9987-y

ILAR (1996). Guide for the Care and Use of Laboratory Animals [EB/OL]. Washington, DC: National Academy Press. Available at: http://oacu.od.nih.gov/ regs/guide/guide.pdf

Kamaraj, C., Rahuman, A. A., Elango, G., Bagavan, A., and Zahir, A. A. (2011). Anthelmintic activity of botanical extracts against sheep gastrointestinal nematodes, Haemonchus contortus. Parasitol. Res. 109, 37-45. doi: 10.1007/ s00436-010-2218-y

Karim, M., Jabeen, K., Iqbal, S., and Javaid, A. (2017). Bioefficacy of a common weed Datura metel against Colletotrichum gloeosporioides. Planta Daninh 35:e017164676.

Kuang, H. X., Yang, B. Y., Tang, L., Xia, Y. G., and Dou, D. Q. (2009). Baimantuoluosides A-C, three new withanolide glucosides from the flower of Datura metel L. Helv. Chim. Acta 92, 1315-1323. doi: 10.1002/hlca.200800404

Kuang, H. X., Yang, B. Y., Xia, Y. G., and Feng, W. S. (2008). Chemical constituents from the flower of Datura metel L. Arch. Pharm. Res. 31, 1094-1097. doi: 10.1007/s12272-001-1274-6

Ma, C. Y., Williams, I. D., and Che, C. T. (1999). Withanolides from Hyoscyamus niger seeds. J. Nat. Prod. 62, 1445-1447.

Mai, N. T., Cuc, N. T., Anh, H. L. T., Nhiem, N. X., Tai, B. H., Yen, P. H., et al. (2017). Two new guaiane sesquiterpenes from Datura metel L. with antiinflammatory activity. Phytochem. Lett. 19, 231-236. doi: 10.1016/j.phytol. 2017.01.011

Murthy, B. K., Nammi, S., Kota, M. K., Rao, R. V. K., Rao, N. K., and Annapurna, A. (2004). Evaluation of hypoglycemic and antihyperglycemic effects of Datura metel (Linn.) seeds in normal and alloxan-induced diabetic rats. J. Ethnopharmacol. 91, 95-98. doi: 10.1016/j.jep.2003.12.010

Pharmacopoeia Commission (2015). Pharmacopoeia of the People's Republic of China, Vol. I. Beijing: Chinese Medical Science and Technology Press, 267.

Rischer, H., Oresic, M., Seppanen-Laakso, T., Katajamaa, M., Lammertyn, F., Ardiles-Diaz, W., et al. (2006). Gene-to-metabolite networks for terpenoid indole alkaloid biosynthesis in Catharanthus roseus cells. Proc. Natl. Acad. Sci. U.S.A. 103, 5614-5619. doi: 10.1073/pnas.0601027103

Roy, S., Samant, L., Ganjhu, R., Mukherjee, S., and Chowdhary, A. (2018). Assessment of in vivo antiviral potential of Datura metel Linn. extracts against rabies virus. Pharmacognosy Res. 10, 109-112. doi: 10.4103/pr.pr_121_17
Sanchez-Gonzalez, M., Lozano-Mena, G., Parra, A., Juan, M. E., and Planas, J. M. (2015). Identification in rat plasma and urine by linear trap quadrupole-orbitrap mass spectrometry of the metabolites of maslinic acid, a triterpene from olives. J. Agr. Food Chem. 63, 1126-1132. doi: 10.1021/jf50 $5379 \mathrm{~g}$

van der Fits, L., and Memelink, J. (2000). ORCA3, a jasmonate-responsive transcriptional regulator of plant primary and secondary metabolism. Science 289, 295-297. doi: 10.1126/science.289.5477.295

Wannang, N. N., and Ndukwe, H. C. (2009). Evaluation of the analgesic properties of the Datura metel seeds aqueous extract. J. Med. Plants Res. 4, 192-195.

Xu, S. L., Liu, Y., Xiang, L., Zhou, F., Li, H. Y., Su, Y. J., et al. (2018). Metabolites identification of bioactive compounds daturataturin A, daturametelin I, n-trans-feruloyltyramine, and cannabisin F from the seeds of Datura metel in rats. Front. Pharmacol. 9:731. doi: 10.3389/fphar.2018. 00731

Yang, B. Y., Xia, Y. G., Liu, Y., Li, L., Jiang, H., Yang, L., et al. (2014). New antiproliferative and immunosuppressive withanolides from the seeds of Datura metel. Phytochem. Lett. 8, 92-96. doi: 10.1016/j.phytol.2014. 02.008

Yang, B. Y., Xia, Y. G., Wang, Q. H., Dou, D. Q., and Kuang, H. X. (2010a). Baimantuoluosides D-G, four new withanolide glucosides from the Flower of Datura metel L. Arch. Pharm. Res. 33, 1143-1148. doi: 10.1007/s12272-0100802-4

Yang, B. Y., Xia, Y. G., Wang, Q. H., Dou, D. Q., and Kuang, H. X. (2010b). Two new amide alkaloids from the flower of Datura metel L. Fitoterapia 81, 1003-1005. doi: 10.1016/j.fitote.2010.06.017

Zhang, H., Bazzill, J., Gallagher, R. J., Subramanian, C., Grogan, P. T., Day, V. W., et al. (2018). Antiproliferative withanolides from Datura wrightii. Planta Med. $79,861-862$.

Ziegler, J., and Facchini, P. J. (2008). Alkaloid biosynthesis: metabolism and trafficking. Annu. Rev. Plant Biol. 59, 735-769. doi: 10.1146/annurev.arplant. 59.032607.092730

Conflict of Interest Statement: The authors declare that the research was conducted in the absence of any commercial or financial relationships that could be construed as a potential conflict of interest.

Copyright (C) 2019 Xia, Liu, Qi, Niu, Zhu, Lu, Xu, Su, Yang and Wang. This is an open-access article distributed under the terms of the Creative Commons Attribution License (CC BY). The use, distribution or reproduction in other forums is permitted, provided the original author(s) and the copyright owner(s) are credited and that the original publication in this journal is cited, in accordance with accepted academic practice. No use, distribution or reproduction is permitted which does not comply with these terms. 